\title{
HEPATIC ARTERIAL INFUSION CHEMOTHERAPY FOR LIVER METASTASIS
}

\author{
Masatoshi Ishizaki ${ }^{1)}$, Norio Akiyama ${ }^{1)}$, Shigebumi Tanaka ${ }^{1)}$, \\ Masahiko Motegi ${ }^{1)}$, Hajime Sasamoto ${ }^{1)}$, Hidenobu Osawa ${ }^{1)}$, \\ Wataru Wada ${ }^{1)}$, Masanobu Nakajima ${ }^{1)}$, Toshihide Iijima ${ }^{2)}$, \\ Masaaki Nemoto ${ }^{3)}$ and Kimitaka Kogure ${ }^{3)}$ \\ 1) Department of Surgery, Public Fujioka General Hospital \\ 2) JR Medical Center \\ 3) 1st Department of Surgery, Gunma University School of Medicine
}

\begin{abstract}
We clinically examined 56 patients with metastatic liver cancer who received hepatic arterial infusion chemotherapy via a reservoir at the Department of Surgery, Public Fujioka General Hospital between January 1989 and March 1999. There were 28 males and 28 females. Patient ages ranged from 40 to 83 years, with a mean of 64.1 years. Primary cancers consisted of colorectal cancer in 34 patients, gastric cancer in 8 patients, gallbladder cancer in 4 patients, breast cancer in 4 patients, leiomyosarcoma in 2 patients, pancreatic cancer in 1 patient, bile duct cancer in 1 patient, ampullary carcinoma in 1 patient and ovarian cancer in 1 patient. Thirty-one patients had simultaneous lesions, while 25 patients had metachronal lesions. A catheter was inserted under laparotomy in 25 patients. A catheter was percutaneously inserted through the femoral artery in 31 patients. Agents included 5-fluorouracil (5-FU), epirubicin hydrochloride (EPI) and mitomycin-C (MMC). There were no serious complications.

In 42 patients who received hepatic arterial infusion chemotherapy excluding patients who underwent surgery for metastatic liver cancer and patients who died of other diseases, the mean survival was 421.1 days. When response was evaluated, complete response (CR) was achieved in 3 patients, partial response (PR) in 12 patients, no change (NC) in 11 patients and progressive disease (PD) in 16 patients. The response rate was $35.7 \%$. One-year, 2-year and 3-year cumulative survival rates were 59\%, 23\% and $8 \%$, respectively. These percentages in the hepatic arterial infusion chemotherapy group were higher than cumulative survival rates in the systemic chemotherapy group and the untreated group. Therefore, hepatic arterial infusion chemotherapy may prolong survival.
\end{abstract}

Key words : Arterial infusion chemotherapy, Reservoir, Liver metastasis

(Kitakanto Med.J. 50 (2) : 111 115, 2000)

\section{INTRODUCTION}

Recent advances in diagnostic imaging procedures have facilitated early detection of metastatic liver cancer. However, there are only a few patients in whom radical hepatectomy is possible. In many patients, metastatic liver cancer is detected as multiple cancer or a portion of systemic metastasis. Therefore, to prolong survival and improve the quality of life (QOL), multidisciplinary treatment is performed. To achieve potentiation of chemotherapy and improve the QOL, we inserted a catheter into the hepatic artery of patients in whom metastatic liver cancer was considered to be a prognostic factor, and administered hepatic arterial infusion chemotherapy using a subcutaneously implanted arterial infusion instrument (reservoir). In this study, we examined the effects of hepatic arterial infusion chemotherapy with a reservoir and complications.

Received : December 6, 1999

Address: MASATOSHI ISHIZAKI, MD

Department of Surgery, Public Fujioka General Hospital

942-1 Fujioka, Fujioka, Gunma 375-8503, Japan 


\section{PATIENTS AND METHODS}

Of 81 patients who received hepatic arterial infusion chemotherapy with a reservoir at the Department of Surgery, Public Fujioka General Hospital between January 1989 and March 1999, this study included 56 patients with metastatic liver cancer. Hepatic arterial infusion chemotherapy with a reservoir was indicated for patients in whom hepatic metastatic foci were considered to be an important prognostic factor excluding patients with cancerous peritonitis. A $5 \mathrm{Fr}$ or 6 Fr Anthron indwelling catheter (Tohre Medical) was used. A Bard Port reservoir (Bard Inc.) was used. Concerning the insertion method, a catheter was inserted through the gastroduodenal artery during laparotomy, and the end of the catheter was fixed in the hepatic artery. Percutaneously, a catheter was inserted into the hepatic artery via the femoral artery. Concerning the administration method, 1000 to $1500 \mathrm{mg} /$ body of 5-FU was administered every week to patients with liver metastasis from colorectal cancer. Furthermore, $250 \mathrm{mg} / \mathrm{qw}$ of $5-\mathrm{FU}, 2 \mathrm{mg} / 2 \mathrm{qw}$ of $\mathrm{MMC}$ and $45 \mathrm{mg} /$ 4qw of EPI were administered to patients with liver metastases from gastric cancer, gallbladder cancer, pancreatic cancer, breast cancer and others, if possible. Results were compared among a group receiving hepatic arterial infusion chemotherapy with a reservoir (hepatic arterial infusion group), a group receiving systemic chemotherapy for liver metastasis during the same period (systemic chemotherapy group) and an untreated group. The cumulative survival rates were compared using Kaplan-Meier's method.

\section{RESULTS}

Patients background: In 56 patients with metastatic liver cancer (28 males, 28 females), hepatic arterial infusion chemotherapy with a reservoir was administered between January 1989 and March 1999. Patient ages ranged from 40 to 83 years, with a mean of 64.1 years. In these patients with metastatic liver cancer, primary foci consisted of colorectal cancer in 34 patients, gastric cancer in 8 patients, gallbladder cancer in 4 patients, breast cancer in 4 patients, gastroduodenal leiomyosarcoma in 2 patients, pancreatic cancer in 1 patient, bile duct cancer in 1 patient, ampullary carcinoma in 1 patient and ovarian cancer in 1 patient (Table 1). Thirty-one patients had simultaneous liver metastases, while 25 patients had metachronal liver metastases. Concerning the insertion method, the catheter was inserted during laparotomy in 25 patients, and percutaneously inserted through the femoral artery in 31 patients.

Metastatic liver cancer (H-No) : In 42 patients who
Table 1. Primary tumor of the liver metastases.

\begin{tabular}{lcc}
\hline Primary tumor & No. of patients & No. of hepatectomy \\
\hline Colorectal ca. & 34 & 10 \\
Gastoric ca. & 8 & 1 \\
Gallbladder ca. & 4 & \\
Breast ca. & 4 & 1 \\
Leiomyosarcoma & 2 & 1 \\
Pancreatic ca. & 1 & \\
Bile duct ca. & 1 & \\
Ampullary ca. & 1 & 13 \\
Ovarian ca. & 1 & \\
\hline Total & 56 &
\end{tabular}

Table 2. H-number of the liver metastases.

\begin{tabular}{ccc}
\hline H-No. & No. of patients & $\mathbf{( \% )}$ \\
\hline H1 & 8 & 19.0 \\
H2 & 18 & 42.9 \\
H3 & 16 & 38.1 \\
\hline
\end{tabular}

Table 3. Response rate in hepatic arterial infusion chemotherapy.

\begin{tabular}{ccc} 
apy. & & \\
\hline Response & No. of patients & (\%) \\
\hline CR & 3 & 7.1 \\
PR & 12 & 28.6 \\
NC & 11 & 26.2 \\
PD & 16 & 38.1 \\
\hline
\end{tabular}

received hepatic arterial infusion chemotherapy with a reservoir excluding patients who underwent surgery for metastatic liver cancer and patients who died of other diseases, $\mathrm{H}-\mathrm{No}$ was evaluated as $\mathrm{H} 1$ in 8 patients $(19.0 \%), \mathrm{H} 2$ in 18 patients $(42.9 \%)$ and $\mathrm{H} 3$ in 16 patients $(38.1 \%)$. A high proportion of patients had multiple cancer lesions involving the bilateral lobes (Table 2).

Treatment response: Using the criteria for direct response to chemotherapy for solid cancer, response was evaluated by computed tomography (CT). CR, PR, NC and PD were achieved in 3 patients (7.1\%), 12 patients $(28.6 \%), 11$ patients $(26.2 \%)$ and 16 patients $(38.1 \%)$ in the hepatic arterial infusion group, respectively. The response rate was $35.7 \%$. The response rate including NC was $61.9 \%$ (Table 3 ).

Prognosis : In the hepatic arterial infusion group, the longest survival was 1181 days. The mean survival was 421.1 days. Furthermore, in the hepatic arterial infusion group, the mean survival was 600.1 days in patients with CR or PR and 334.9 days in patients with PD or NC. In the systemic chemotherapy group, the longest survival was 1173 days, with a mean of 218.1 days. In the untreated group, the longest survival was 330 days, with a mean of 97.6 days (Table 4). In the hepatic arterial infusion group, 1-year, 2-year and 3 -year cumulative survival rates were $59 \%, 23 \%$ and 
Hepatic arterial infusion chemotherapy

Table 4. Comparison of the survival time after treatment with hepatic arterial infusion chemotherapy, systemic chemotherapy and untreated group.

\begin{tabular}{lcc}
\hline Treatment & Longest survival time (days) & Mean survival time (days) \\
\hline Hepatic arterial infusion chemotherapy & 1181 & 421.1 \\
$(\mathrm{CR}+\mathrm{PR})$ & & $(600.1)$ \\
(PD+NC) & & $(334.9)$ \\
Systemic chemotherapy & 1173 & 218.1 \\
Untreated & 330 & 97.6 \\
\hline
\end{tabular}

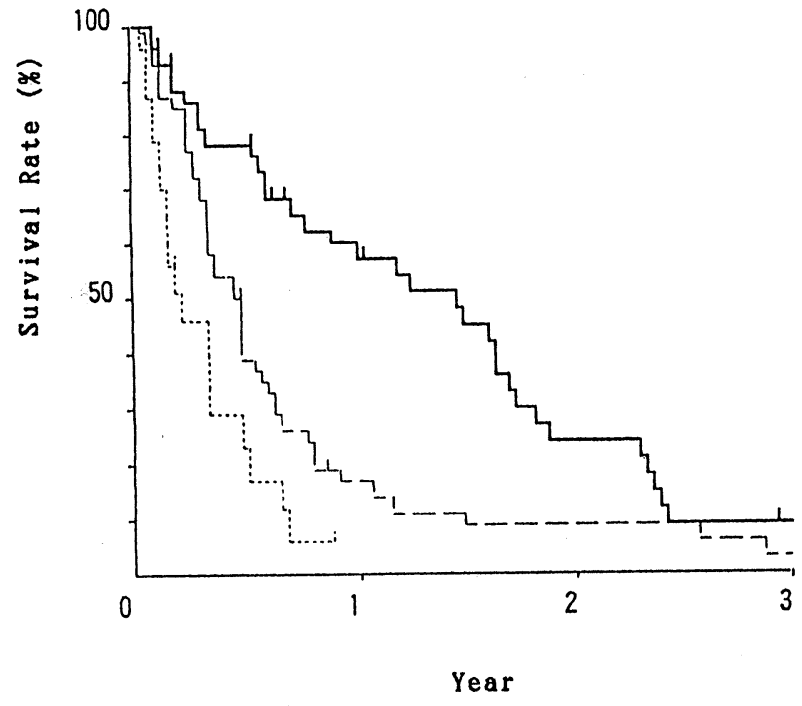

Fig. 1. Survival curves of the patients with liver metastases treated by hepatic arterial infusion chemotherapy (solid line), systemic chemotherapy (dashed line) and untreated group (dotted line).

$8 \%$, respectively. In the systemic chemotherapy group, 1-year, 2-year and 3-year cumulative survival rates were $16 \%, 8 \%$ and $2 \%$, respectively. In the untreated group, none of the patients achieved 1-year survival (Fig. 1). Furthermore, in the hepatic arterial infusion group, 1-year, 2-year and 3-year cumulative survival rates were $91 \%, 45 \%$ and $18 \%$ in patients with CR or $\mathrm{PR}$ as well as $41 \%, 12 \%$ and $4 \%$ in patients with PD or NC, respectively (Fig. 2).

Complications: Complications associated with the reservoir system included catheter occlusion in 11 patients (19.6\%), hemorrhage in 2 patients (3.6\%), infection in 2 patients $(3.6 \%)$ and intraduodenal migration in 1 patient $(1.9 \%)$. Furthermore, drug administration-associated complications included nausea and vomiting in 8 patients (14.4\%), leukopenia in 6 patients $(10.7 \%)$, epigastralgia in 2 patients $(3.6 \%)$ and gastroduodenal ulcer in 1 patient (1.9\%). Four patients required hospitalization due to complications. In 8 patients, treatment was discontinued due to complications. Furthermore, in 5 patients, an additional catheter was inserted to continue treatment (Table 5).

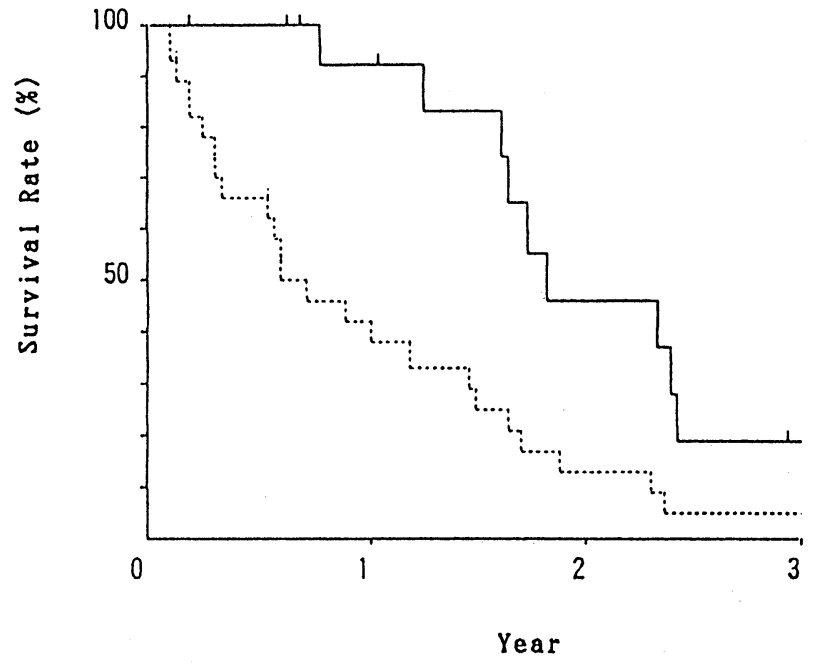

Fig. 2. Survival curves of the patients with liver metastases treated by arterial infusion chemotherapy $(\mathrm{CR}+\mathrm{PR}$ : solid line and PD $+\mathrm{NC}$ : dotted line).

Table 5. Complications of hepatic arterial infusion chemotherapy.

\begin{tabular}{lc}
\hline Complications & No. of patients (\%) \\
\hline Catheter occlusion & $11(19.6 \%)$ \\
Hemorrhage & $2(3.6 \%)$ \\
Infection & $2(3.6 \%)$ \\
Intraduodenal migration & $1(1.9 \%)$ \\
\hline Nausea/vomiting & $8(14.4 \%)$ \\
Leukopenia $<3000$ & $6(10.7 \%)$ \\
Epigastralgia & $2(3.6 \%)$ \\
Gastroduodenal ulcer & $1(1.6 \%)$ \\
\hline
\end{tabular}

\section{DISCUSSION}

Metastatic liver cancer is an important factor influencing prognosis. In recent years, hepatectomy has been aggressively performed to treat metastatic liver cancer. However, resection is still impossible in many patients. This is because most patients with metastatic liver cancer have multiple lesions and because most metastatic liver cancer lesions are part of systemic metastases. In such patients, hepatic arterial infusion chemotherapy via a reservoir has been performed, and the usefulness of this procedure has been reported ${ }^{1 \sim 5)}$. With respect to the advantage of this procedure, hepatic arterial infusion can maintain a relatively higher 
drug concentration in tumor tissues than that in normal liver tissues, thus relieving systemic side effects, since metastatic liver cancer is mainly nourished through the hepatic artery ${ }^{6}$. Furthermore, this procedure is also significant for improving the QOL, since treatment can be continued at the Outpatient Clinic ${ }^{7)}$. However, some studies have indicated that this procedure dose not contribute to prolonged survival, although many studies have reported tumor-reducing effects of hepatic arterial infusion chemotherapy ${ }^{8)}$.

Considering the QOL, we administered hepatic arterial infusion chemotherapy via a reservoir, which facilitates treatment at the Outpatient Clinic, to patients in whom hepatectomy would be difficult and metastatic liver cancer was an important prognostic factor even in the presence of other organ or lymph node metastases. In 34 of the patients who received hepatic arterial infusion chemotherapy with a reservoir at our department, the primary foci were colorectal cancer lesions. Colorectal cancer showed the highest percentage, followed by gastric cancer in 8 patients, gallbladder cancer in 4 patients and breast cancer in 4 patients. This is because colorectal cancer and gastric cancer primarily metastasize to the liver via the portal vein in many patients, facilitating early detection and treatment prior to systemic metastasis. However, in many patients with gallbladder cancer, breast cancer, or pancreatic cancer, metastatic liver cancer is part of systemic metastases, and treatment of metastatic liver cancer alone cannot be indicated. We administered a massive dose of 5-FU intermittently to treat metastatic liver cancer from colorectal cancer, and combination therapy with 5-FU, EPI and MMC to treat metastatic liver cancer from gastric cancer, gallbladder cancer, pancreatic cancer, breast cancer and others.

The effects of hepatic arterial infusion chemotherapy with a reservoir on metastatic liver cancer at our department were compared to results in the systemic chemotherapy group and the untreated group during the same period. In the hepatic arterial infusion group, $\mathrm{CR}$ and PR were achieved in 3 and 12 of 42 patients, respectively. The response rate was $35.7 \%$. In the systemic chemotherapy group, neither CR nor PR was achieved in any patient. The mean survival in the hepatic arterial infusion group was 421.1 days, and was slightly prolonged compared to values in the systemic chemotherapy group and the untreated group (218.1 days, 97.6 days). In the hepatic arterial infusion group, 1-year, 2-year and 3-year cumulative survival rates were $59 \%, 23 \%$ and $8 \%$, respectively. These percentages were higher than those in 2 other groups. Furthermore, in patients with $\mathrm{CR}$ or PR in the hepatic arterial infusion group, the mean survival was prolonged and cumulative survival rates were increased compared to those in patients with PD or NC. Since the systemic chemotherapy group and the untreated group included patients in whom factors other than metastatic liver cancer were prognostic factors, it may be inappropriate to uniformly compare these 3 groups. However, in the hepatic arterial infusion group, survival was prolonged and survival rates were increased compared to those in 2 other groups, suggesting the usefulness of hepatic arterial infusion chemotherapy. However even if liver metastasis is controlled by hepatic arterial infusion chemotherapy, liver metastasis or extrahepatic lesions may deteriorate after hepatic arterial infusion chemotherapy is discontinued, showing a poor prognosis. Therefore, hepatic arterial infusion should be administered for a prolonged period if possible, or extrahepatic lesions must be controlled.

Concerning complications, the incidence of catheter or port-associated complications was $28.7 \%$. The incidence of drug-related side effects was $30.6 \%$. However, there were no serious complications. Complications can be prevented by skilled procedure and treatment $t^{9}$. Since hepatic arterial infusion chemotherapy via a reservoir can be managed by the Outpatient Clinic, this procedure is readily accepted by patients, and may be useful. In the future, agents for hepatic arterial infusion chemotherapy should be selected, and therapeutic strategies for extrahepatic lesions should be established.

\section{CONCLUSIONS}

To treat metastatic liver cancer, we administered hepatic arterial infusion chemotherapy via a reservoir. In the hepatic arterial infusion group, CR was achieved in $7.1 \%$ of patients, PR in $28.6 \%$ of patients, NC in $26.2 \%$ of patients and PD in $38.1 \%$ of patients. The response rate was $35.7 \%$. The mean survival was 421.1 days. One-year, 2-year and 3-year cumulative survival rates were $59 \%, 23 \%$ and $8 \%$, respectively. In patients with CR or PR in the hepatic arterial infusion group, the mean survival was 600.1 days. One-year, 2 -year and 3-year cumulative survival rates were $91 \%$, $45 \%$ and $18 \%$, respectively, showing favorable results. Furthermore, in patients with PD or NC, the mean survival was 334.9 days. One-year, 2-year and 3-year cumulative survival rates were $41 \%, 12 \%$ and $4 \%$, respectively.

These results were better than those in the systemic chemotherapy group and the untreated group. Hepatic arterial infusion chemotherapy via a reservoir may prolong survival, while maintaining QOL, since treatment at the Outpatient Clinic is possible. 


\section{REFERENCES}

1) Seki H, Kimura M, Yoshimura N, et al. Hepatic arterial infusion chemotherapy with 5fluorouracil-based regimens in the management of liver metastases of colorectal carcinoma. Int $\mathrm{J}$ Clin Oncol 1998; 3 : 171-175.

2) Emmoto T, Ohsawa J, Nakanishi M, et al. Study of hepatic arterial infusion chemotherapy for colorectal cancer patients with synchronous liver metastases. Nippon Rinsho Geka Igakkai Zasshi (J Jpn Soc Clin Surg) 1993; 54 : 2993-3001.(in Japanese)

3) Nonami T, Hashimoto M, Yasui M, et al. Therapeutic effect and prognostic factors in patients with unresectable liver metastasis from colorectal cancer treated by hepatic artery chemotherapy. Nippon Gan Chiryo Gakkaishi (J Jpn Soc Cancer Ther) 1994 ; 29 : 1-6.(in Japanese)

4) Sugihara K, Moriya $Y$ and Akasu T. Hepatic arterial infusion therapy for unresectable colorectal liver metastases. Nippon Daicho Komonbyo Gakkai Zasshi (J Jpn Soc Coloproctal) 1994 ; 47 : 1127-1133.(in Japanese)

5) Uemura T, Kawasaki S, Kawai H, et al. Evalua- tion of hepatic arterial infusion chemotherapy for gastric cancer. Gan to Kagakuryoho (Jpn J Cancer Chemother) 1996;23:1787-1791.(in Japanese)

6) Okuno $\mathrm{K}$ and Yasutomi $\mathrm{M}$. Hepatic arterial infusion for liver metastases from colorectal cancer. Rinsho Geka (J Clin Surg) 1999; 54 : 171179.(in Japanese)

7) Takahashi M, Nakano S, Takeda I, et al. The clinical evaluation of hepatic arterial infusion chemotherapy in patients with liver metastases from colorectal cancer employing an implanted port system. Nippon Shokakibyo Gakkai Zasshi (Jpn J Gastroenterol) 1996;93: 10-18.(in Japanese)

8) Kawahara H, Hirai K, Ashikaga K, et al. Analysis of colorectal cancers with liver metastasis. Nippon Daicho Komonbyo Gakkai Zasshi (J Jpn Soc Coloproctal) 1993; $46: 746-750$.(in Japanese)

9) Aoki T, Inoue K, Tsuchida A, et al. A study of various complications in arterial infusion chemotherapy. Gan to Kagakuryoho (Jpn J Cancer Chemother) $1994 ; 21: 2649-2654$.(in Japanese) 